\title{
Article \\ A Model of Directed Graph Cofiber
}

\author{
Zachary McGuirk ${ }^{1}$ and Byungdo Park ${ }^{2, *}$
}

1 Einstein Institute of Mathematics, Edmond J. Safra Campus, The Hebrew University of Jerusalem, Jerusalem 91904, Israel; zachary.mcguirk@mail.huji.ac.il

2 Department of Mathematics Education, Chungbuk National University, Cheongju 28644, Korea

* Correspondence: byungdo@chungbuk.ac.kr

\begin{abstract}
In the homotopy theory of spaces, the image of a continuous map is contractible to a point in its cofiber. This property does not apply when we discretize spaces and continuous maps to directed graphs and their morphisms. In this paper, we give a construction of a cofiber of a directed graph map whose image is contractible in the cofiber. Our work reveals that a category-theoretically correct construction in continuous setup is no longer correct when it is discretized and hence leads to look at canonical constructions in category theory in a different perspective.
\end{abstract}

Keywords: directed graphs; graph homotopy; cofiber; category theory; path-space homology; combinatorics

MSC: primary 55P99; secondary 05C20; $18 \mathrm{~A} 30$

\section{Introduction}

Directed graphs are everywhere. They are fundamental mathematical objects not only for mathematical interests but also for applications including electric circuits, neural networks, social relationships, etc. [1,2]. Naturally classifying directed graphs through algebraic invariants is an important problem, and there have been various attempts (see [3] and references therein). Among them, a recent development of path-space homology and cohomology for directed graphs by Grigor'yan, Lin, Muranov, and Yau [3-5] is of particular interest. Their constructions are not too trivial, not too hard to compute, can be non-trivial for degrees greater than one, and satisfy similar properties to those invariants in the continuous and smooth setup. It turns out that path-space homology satisfies discrete analogs of Eilenberg-Steenrod axioms [6], i.e., the Künneth formula [7]; it is generalized to multigraphs and quivers [8] and has applications to discretizing Morse theory [9].

An interesting result regarding the path space homology is that it satisfies homotopy invariance. The history of homotopy theory for directed graphs goes back to $[10,11]$ and more recently to [12]. In 2014, Grigor'yan, Lin, Muranov, and Yau [13] considered digraph homotopy as a notion of a discrete analog of homotopy in topology and proved that their path-space homology is invariant under this version of homotopy. Indeed, their homotopy theory for directed graphs is a natural "discretization" that is leading to establishing a dictionary between homotopy theory of spaces and its discrete counterpart. For example, several theorems in classical homotopy theory, such as van Kampen theorem, Hurewicz theorem, and Brown representability theorem [14,15], are successfully discretized (see $[13,16])$, and model structures in the category of directed graphs and related questions are currently being investigated [17].

From the perspective of homotopy theory and category theory, the homotopy theory for directed graphs is an interesting source to understand existing classical results. Any time a classical construction or theory fails to be discretized or satisfy expected properties, the failure itself or remedies to it reveal the essence of the classical theory. For example, the path-space homology satisfies all discrete analogs of Eilenberg-Steenrod axioms, but these 
axioms are not complete (see [6]), and we note that the completeness of Eilenberg-Steenrod axioms has a topological nature in it. Another example is a failure of homotopy extension property for directed graphs [16], which reveals that the Brown representability theorem for not-necessarily-finite $\mathrm{CW}$ complexes is likely to be a special feature of CW complexes rather than a consequence of generalities in category theory.

In [16], the authors have proven Brown representability theorem for finite directed graphs by adopting the methodology of J. F. Adams [18]. The gist of technical innovation was the use of digraph cofibers and mapping tubes (see [16] (Definitions 2.21 and 2.25)). It means that a natural discretization of Adams' proof itself does not yield Brown representability theorem, but by appropriately modifying category-theoretic and homotopytheoretic constructions, one can obtain this result. Accordingly, searching for constructions in directed graphs that satisfy general homotopy-theoretic properties lies at the technical core of discretizing classical results.

This paper is a brief note on a possible construction of a cofiber of directed graph map satisfying the property that the image of the directed graph map can be homotoped to a point. A cofiber of continuous map in the category of spaces satisfies this property in an obvious manner. However, in the category of directed graphs, any time there is an edge from a vertex in the range of the directed graph map to a vertex outside, it prevents defining a directed graph homotopy. We have constructed a model of cofiber that is free from such issues.

This paper is organized as follows. Section 2 collects preliminaries to work in the category of directed graphs. Sections 3 and 4 review necessary operations in the category of directed graphs and homotopy theory for directed graphs. In Section 5, we give a construction of a directed graph cofiber proposed above and prove that it satisfies the desired property.

\section{The Category of Directed Graphs}

In this section, we shall review basic definitions and notations comprising the category of directed graphs.

Definition 1 ([13]). A directed graph (or digraph for short) $\vec{G}$ is a pair $(V, E)$ consisting of a set of vertices $V$ and a set $E$ of ordered pairs of distinct vertices in $V$ called edges.

Note that our digraphs do not allow loop-edges or multiple edges with the same source and target. A point is a digraph $(V, E)$ with $V$ a singleton and $E$ an empty set.

Definition 2. Let $n \in \mathbb{Z}^{+}$. An n-step line digraph is a digraph consisting of vertices labeled by $0,1, \cdots, n$ and exactly one edge at every two consecutive vertices, i.e., either $(i-1, i)$ or $(i, i-1)$ for all $i \in \mathbb{Z}^{+}$such that $1 \leq i \leq n$.

An $n$-step line digraph is also called a path digraph or a linear digraph. When $n=1$, there are two possible line digraphs, $I^{+}:=0 \rightarrow 1$ and $I^{-}:=0 \leftarrow 1$.

Notation 1. We will denote by $I_{n}$ an arbitrary n-step line digraph and by $\mathcal{J}_{n}$ the totality of all possible $n$-step line digraphs. The set of all line digraphs of any length will be denoted by $\mathcal{J}=\cup_{n} \mathcal{J}_{n}$.

Definition 3. A digraph map, $f: \vec{G} \rightarrow \vec{H}$, is a function from the vertex set of $\vec{G}$ to the vertex set of $\vec{H}$ such that whenever $(x, y)$ is an edge in $\vec{G}$ either $f(x)=f(y)$ in $\vec{H}$ or $(f(x), f(y))$ is an edge in $\vec{H}$.

Definition 4. The image digraph $\operatorname{Im}(f)$ of a digraph map $f: \vec{G} \rightarrow \vec{H}$ is a directed graph consisting of avertex set $f\left(V_{\vec{G}}\right)$ and an edge set $E_{\vec{G}}=\left\{(f(x), f(y)): x, y \in V_{\vec{G}^{\prime}} f(x) \neq f(y)\right.$, and $\left.(x, y) \in E_{\vec{G}}\right\}$.

Definition 5 . The category of directed graphs $\mathcal{D}$ is a category in which the objects are directed graphs, $\vec{G}$, and the morphisms are digraph maps, $f: \vec{G} \rightarrow \vec{H}$. 


\section{Operations in Directed Graphs}

In this section, we review operations in directed graphs. The definition of an identification digraph is central in yielding homotopy-theoretic constructions in the category of directed graphs.

Definition 6. A sub-digraph $\vec{X}$ of a digraph $\vec{G}$ denoted $\vec{X} \subset \vec{G}$ is a digraph for which $V_{\vec{X}} \subset V_{\vec{G}}$ and $E_{\vec{X}} \subset E_{\vec{G}}$.

Note that even if $u, v \in V_{\vec{X}}$ and $(u, v) \in E_{\vec{G}^{\prime}}$ it is not necessarily the case that $(u, v) \in E_{\vec{X}}$.

Definition 7. An induced sub-digraph $\vec{X}$ of a digraph $\vec{G}$ denoted $\vec{X} \sqsubset \vec{G}$ is a sub-digraph in which whenever $u, v \in V_{\vec{X}}$ and $(u, v) \in E_{\vec{G}}$, then $(u, v) \in E_{\vec{X}}$ as well.

Definition 8. The vertex boundary of a sub-digraph $\vec{X} \subset \vec{G}$ is

$$
\partial \vec{X}:=\left\{g \in V_{\vec{G}} \backslash V_{\vec{X}} \mid \text { either }(g, x) \in E_{\vec{G}} \text { or }(x, g) \in E_{\vec{G}} \text {, where } x \in V_{\vec{X}}\right\} .
$$

Definition 9. The intersection of digraphs $\vec{G}$ and $\vec{H}$, denoted by $\vec{G} \cap \vec{H}$, is the digraph consisting of $V_{\vec{G} \cap \vec{H}}=V_{\vec{G}} \cap V_{\vec{H}}$ and $E_{\vec{G} \cap \vec{H}}=E_{\vec{G}} \cap E_{\vec{H}}$.

Definition 10. The union of digraphs $\vec{G}$ and $\vec{H}$, denoted by $\vec{G} \cup \vec{H}$, is the digraph consisting of $V_{\vec{G} \cup \vec{H}}=V_{\vec{G}} \cup V_{\vec{H}}$ and $E_{\vec{G} \cup \vec{H}}=E_{\vec{G}} \cup E_{\vec{H}}$.

Definition 11. The disjoint union of two digraphs $\vec{G}$ and $\vec{H}$, denoted $\vec{G} \amalg \vec{H}$ is given by the disjoint union of their respective vertex sets and edge sets, as sets.

Definition 12. The graph Cartesian product $\square$ of two directed graphs $\vec{G}$ and $\vec{H}$ is a directed graph $\vec{G} \square \vec{H}$, where the vertices are all ordered pairs $(u, v)$ such that $u \in V_{\vec{G}}$ and $v \in V_{\vec{H}}$, and $\left(u_{1}, v_{1}\right) \rightarrow\left(u_{2}, v_{2}\right)$ is an edge in $\vec{G} \square \vec{H}$ if either $u_{1}=u_{2}$ and $v_{1} \rightarrow v_{2}$ in $\vec{H}$, or $u_{1} \rightarrow u_{2}$ in $\vec{G}$ and $v_{1}=v_{2}$.

Remark 1. Given a fixed vertex $v_{0} \in V_{\vec{H}^{\prime}}$ we will denote by $\vec{G} \square\left\{v_{0}\right\}$ the $v_{0}$-slice of $\vec{G} \square \vec{H}$. It is the induced sub-digraph where the vertices are all ordered pairs $\left(u, v_{0}\right)$ such that $u \in V_{\vec{G}}$ and the edges are those resulting from the edges of $\vec{G}$.

Definition 13. Let $\vec{G}$ and $\vec{H}$ be digraphs and $\sim$ an equivalence relation on vertex sets of $\vec{G}$ and $\vec{H}$ such that whenever $\left(g_{1}, g_{2}\right) \in E_{\vec{G}^{\prime}} g_{1} \sim h_{1}$, and $g_{2} \sim h_{2}$, then either $h_{1}=h_{2}$ or $\left(h_{1}, h_{2}\right) \in E_{\vec{H}}$. The identification digraph resulting from the relation $\sim$ is a digraph $\vec{G} \amalg \vec{H} / \sim$ whose vertices are equivalence classes and whose edges are the edges between the representatives of the classes.

Definition 14. A quotient digraph $\vec{G} / \vec{X}$, for $\vec{X} \subset \vec{G}$ and $\vec{X}$ not necessarily connected, is an identification digraph $\vec{G} \amalg * / \sim$ where $x \sim *$ for all $x \in V_{\vec{X}}$.

\section{Homotopy for Digraphs}

In this section, we review the notion of homotopy a là Grigor'yan, Lin, Muranov, and Yau [13].

Definition 15. Two digraph maps $f, g: \vec{G} \rightarrow \vec{H}$ are homotopic, denoted $f \simeq g$, if there exists an $n \geq 1$ and a digraph map $F: \vec{G} \square I_{n} \rightarrow \vec{H}$, for some line digraph $I_{n} \in \mathcal{J}_{n}$ (recall Notation 1 ), such that $\left.F\right|_{\vec{G} \times\{0\}}=f$ and $\left.F\right|_{\vec{G} \times\{n\}}=g$. 
Definition 16. Two digraphs are said to be homotopically equivalent (or to be of the same homotopy type) if there exist two digraph maps, $g: \vec{G} \rightarrow \vec{H}$ and $h: \vec{H} \rightarrow \vec{G}$, such that $h \circ g \simeq i d_{\vec{G}}$ and $g \circ h \simeq i d_{\vec{H}}$.

Example 1. Let $f: \vec{G} \rightarrow \vec{H}$ be a digraph map. The mapping cylinder $\vec{M}_{f}$ and the digraph $\vec{H}$ are homotopically equivalent. This can be shown by taking a homotopy $F: \vec{M}_{f} \square I^{-} \rightarrow \vec{M}_{f}$ defined by $F(-, 0)=i d_{\vec{M}_{f}} F((g, 0), 1)=(f(g), 1)$ for all $g \in V_{\vec{G}}$ and $F((h, 1), 1)=(h, 1)$ for all $h \in V_{\vec{H}}$.

Definition 17. A digraph $\vec{G}$ is said to be contractible if there exists a homotopy between $i d_{\vec{G}}$ and a constant digraph map.

Definition 18. The homotopy category of directed graphs, denoted HoD, is a category in which the objects are directed graphs and the morphisms are equivalence classes of digraph maps where $f \simeq g$ whenever $f$ and $g$ are homotopic.

\section{Main Construction}

In this section, we give a construction of a digraph cofiber satisfying the property that the image of a digraph map is contractible in the digraph cofiber. To this end, we set up extensions of a mapping cylinder and a cone that enables lifting edges. The main outcome in this paper is Construction 1 , from which Theorem 1 follows.

Definition 19. The mapping cylinder of a digraph map $f: \vec{G} \rightarrow \vec{H}$ is given by

$$
\vec{M}_{f}:=\left[\vec{G} \square I^{-} \coprod \vec{H}\right] / \sim,
$$

where $(g, 0) \sim f(g)$ for all $g \in V_{\vec{G}}$.

Definition 20. An extension of a mapping cylinder $\vec{M}_{f}$ for a digraph map $f: \vec{G} \rightarrow \vec{H}$ is the digraph $\vec{E}_{f}=\left(V_{\vec{E}_{f}}, E_{\vec{E}_{f}}\right)$ where,

$$
V_{\vec{E}_{f}}=V_{\vec{G}} \coprod V_{\vec{H}^{\prime}}
$$

and $(u, v)$ is an edge in $E_{\vec{E}_{f}}$ if $(u, f(v)) \in E_{\vec{H}}$ for some $u \in V_{\vec{H}}$ and $v \in V_{\vec{G}}$ or in the opposite direction $(v, u)$ is an edge if $(f(v), u) \in E_{\vec{H}}$ for some $u \in V_{\vec{H}}$ and $v \in V_{\vec{G}}$.

Definition 21. An extended mapping cylinder for a digraph map $f: \vec{G} \rightarrow \vec{H}$ is given by the digraph $\overrightarrow{E M}_{f}=\vec{M}_{f} \cup \vec{E}_{f}$.

Definition 22. The cone $C \vec{G}$ over a digraph $\vec{G}$ is the digraph $\left[\vec{G} \square I^{-}\right] / \sim$, where $(g, 0) \sim *$ for all $g \in V_{\vec{G}}$.

Definition 23. An extension of a cone over a sub-digraph $\vec{X} \subset \vec{H}$ is the digraph $\vec{B}_{\vec{X}}=\left(V_{\vec{B}_{\vec{X}}}, E_{\vec{B}_{\vec{X}}}\right)$ where $V_{\vec{B}_{\vec{X}}}=\partial \vec{X} \cup\{p\}$, $p$ is the cone point, and $(p, y) \in E_{\vec{B}_{\vec{X}}}$ if and only if $(x, y) \in E_{\vec{H}}$ for some $x \in \vec{X}$ and $y \in \partial \vec{X}$ or $(y, p) \in E_{\vec{B}_{\vec{X}}}$ if and only if $(y, x) \in E_{\vec{H}}$ for some $x \in \vec{X}$ and $y \in \partial \vec{X}$.

Definition 24. An extended cone over the sub-digraph $\vec{X}$ in $\vec{H}$ is $C \vec{X} \cup \vec{B}_{\vec{X}}$.

Construction 1. The digraph cofiber $\vec{C}(f)$ for a map $f: \vec{G} \rightarrow \vec{H}$ is an extension of a cone over $\vec{G} \amalg \operatorname{Im}(f)$ (viewed as an induced subdigraph of $\vec{M}_{f}$ ) unioned with the identification digraph $\vec{G} \square I^{+} \amalg \vec{H} / \sim$ where $(g, 0) \sim f(g)$ and the extension of this reversed mapping cylinder (See Figure 1). 


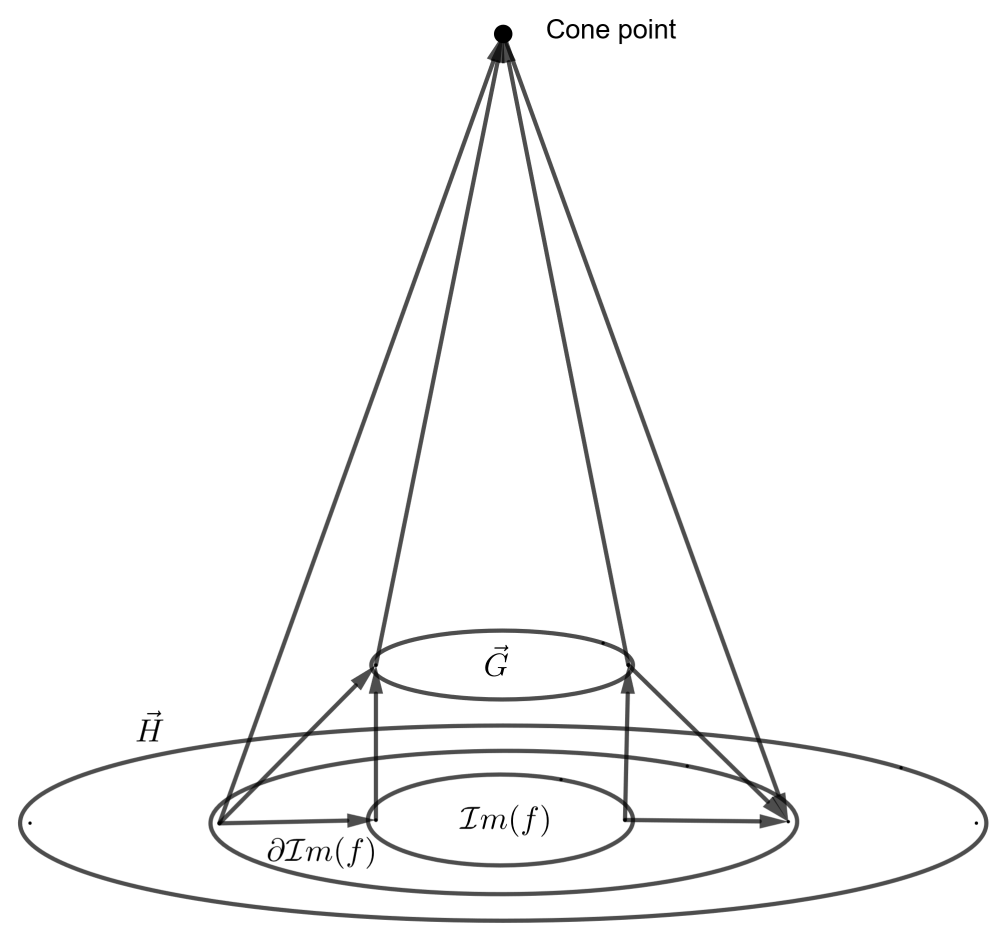

Figure 1. Digraph cofiber of a digraph map $f: \vec{G} \rightarrow \vec{H}$.

Remark 2. The resulting structure in the above definition is a "two-step extended cone" with a middle slice that preserves a copy of $\vec{G}$. It should be noted that $\vec{C}(f)$ is not a category-theoretic cofiber. However, from the perspective of homotopy theory for digraphs, category-theoretic cofibers do not carry much meaning. Specifically, for a digraph map $f: \vec{G} \rightarrow \vec{H}$, note that its category-theoretic cofiber is a pushout of the following diagram in the category $\mathcal{D}$.

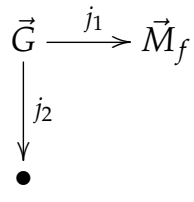

where $\bullet$ is a point, $j_{1}$ the inclusion, and $j_{2}$ the constant map. Not only does the pushout not have a slice of $\vec{G}$ in it, and as mentioned in Section 1, but one cannot homotope $\operatorname{Im}(f)$ to the vertex of the cone. To further develop homotopy theory for digraphs, we have to construct a cofiber that retains essential properties of a cofiber of continuous map, and from the construction above, one can see that our construction retains a copy of the domain digraph $\vec{G}$. We claim that $\operatorname{Im}(f)$ can be homotoped to the cone vertex as stated below.

Theorem 1. Suppose $f: \vec{G} \rightarrow \vec{H}$ is a map of digraphs. Consider the sequence $\vec{G} \stackrel{f}{\rightarrow} \vec{H} \stackrel{i}{\rightarrow} \vec{C}(f)$ where the map $i$ is the inclusion. Then the composition $i$ o $f$ is homotopically trivial.

Proof. We have to prove that the subdigraph $\operatorname{Im}(f)$ is contractible in $\vec{C}(f)$. By construction of $\vec{C}(f)$, we define a homotopy $F_{1}: \vec{C}(f) \square I^{+} \rightarrow \vec{C}(f)$ that takes every $f(g) \in \operatorname{Im}(f)$ for $g \in V_{\vec{G}}$ to $(g, 1)$ in the $\vec{G} \square\{1\}$-slice in $\vec{G} \square I^{+} \amalg \vec{H} / \sim$ and then $F_{2}: \vec{C}(f) \square I^{+} \rightarrow \vec{C}(f)$ that takes this slice to the cone vertex. The existence of digraph maps $F_{1}$ and $F_{2}$ is guaranteed by Definition 23.

\section{Discussion}

In this article, we have constructed a model of a cofiber of a digraph map. A cofiber of a continuous map in the category of spaces loses homotopy-theoretically essential prop- 
erties after being discretized to a category-theoretic cofiber in the category of digraphs. Our construction retains those essential properties, as seen in Section 5. In addition, our work reveals that a universal construction in category theory is not necessarily a homotopytheoretically correct construction when we consider a category consisting of discrete objects. Therefore, further research in this category will provide interesting perspectives for category theory.

Author Contributions: Conceptualization, Z.M. and B.P.; methodology, Z.M. and B.P.; writingoriginal draft preparation, Z.M. and B.P.; writing-review and editing, Z.M. and B.P.; visualization, Z.M.; project administration, Z.M. and B.P.; funding acquisition, B.P. All authors have read and agreed to the published version of the manuscript.

Funding: This work was supported by the National Research Foundation of Korea (NRF) grant funded by the Korea government (MSIT) (No. 2020R1G1A1A01008746) and the research grant of the Chungbuk National University in 2019.

Institutional Review Board Statement: Not applicable.

Informed Consent Statement: Not applicable.

Data Availability Statement: Not applicable.

Acknowledgments: Some of the research that resulted in this paper was carried out in Seoul at Korea Institute for Advanced Study (KIAS) and then later at the Hebrew University of Jerusalem. We thank these institutions for their support and hospitality.

Conflicts of Interest: The authors declare no conflict of interest.

\section{References}

1. Gurevich, B.M. On classes of infinite loaded graphs with randomly deleted edges. Appl. Math. Nonlinear Sci. 2020, 5, 257-260. [CrossRef]

2. Hosamani, S.M.; Awati, V.B.; Honmore, R.M. On graphs with equal dominating and c-dominating energy. Appl. Math. Nonlinear Sci. 2019, 4, 503-512. [CrossRef]

3. Grigor'yan, A.; Lin, Y.; Muranov, Y.; Yau, S.-T. Homologies of path complexes and digraphs. arXiv 2012, arXiv:1207.2834.

4. Grigor'yan, A.; Lin, Y.; Muranov, Y.; Yau, S.-T. Cohomology of digraphs and (undirected) graphs. Asian J. Math. 2015, 19, 887-931. [CrossRef]

5. Grigor'yan, A.; Muranov, Y.; Yau, S.-T. On a cohomology of digraphs and Hochschild cohomology. J. Homotopy Relat. Struct. 2016, 11, 209-230. [CrossRef]

6. Grigor'yan, A.; Jimenez, R.; Muranov, Y.; Yau, S.-T. On the path homology theory of digraphs and Eilenberg-Steenrod axioms. Homol. Homotopy Appl. 2018, 20, 179-205. [CrossRef]

7. Grigor'yan, A.; Muranov, Y.; Yau, S.-T. Homologies of digraphs and Künneth formulas. Comm. Anal. Geom. 2017, 25, 969-1018. [CrossRef]

8. Grigor'yan, A.; Muranov, Y.; Vershinin, V.; Yau, S.-T. Path homology theory of multigraphs and quivers. Forum Math. 2018, 30, 1319-1337. [CrossRef]

9. $\quad$ Lin, Y.; Wang, C.; Yau, S.-T. Discrete Morse Theory on Digraphs. arXiv 2021, arXiv:2102.10518.

10. Gianella, G.M. Su una omotopia regolare dei grafi. Rend. Sem. Mat. Univ. Politec. Torino 1976, 35, 349-360.

11. Malle, G. A homotopy theory for graphs. Glas. Mat. Ser. III 1983, 18, 3-25.

12. Chen, B.; Yau, S.-T.; Yeh, Y.-N. Graph homotopy and Graham homotopy. Discret. Math. 2001, 241, 153-170. [CrossRef]

13. Grigor'yan, A.; Lin, Y.; Muranov, Y.; Yau, S.-T. Homotopy theory for digraphs. Pure Appl. Math. Q. 2014, 10, 619-674. [CrossRef]

14. Brown, E.H., Jr. Cohomology theories. Ann. Math. 1962, 75, 467-484. [CrossRef]

15. Brown, E.H., Jr. Abstract homotopy theory. Trans. Amer. Math. Soc. 1965, 119, 79-85. [CrossRef]

16. McGuirk, Z.; Park, B. Brown representability for directed graphs. arXiv 2021, arXiv:2003.07426.

17. Sampietro, J. Homotopy Theory of Digraphs-A Categorical Viewpoint. MCA Special Session: Categories and Topology. 2021. Available online: https:/ / www.youtube.com/watch?v=C_O92uCk1Qo (accessed on 20 December 2021).

18. Adams, J.F. A variant of EH Brown's representability theorem. Topology 1971, 10, 185-198. [CrossRef] 